\title{
Business and Information System Alignment: a Formal Solution for Telecom Services
}

\author{
Jacques Simonin 1 , Emmanuel Bertin ${ }^{2}$, Yves Le Traon ${ }^{3}$, Jean-Marc Jézéquel ${ }^{4}$, Noël Crespi ${ }^{5}$ \\ ${ }^{1}$ Institut Télécom, Télécom Bretagne, Lab-STICC UMR CNRS 3192, UEB, Technopole Brest-Iroise, 29238 Brest, France \\ 2 Orange Labs, 42 rue des Coutures, 14066 Caen, France \\ 3 Université du Luxembourg, 6, rue Richard Coudenhove-Kalergi, 1359 Luxembourg, Luxembourg \\ 4 INRIA and Rennes University, Campus Universitaire de Beaulieu, 35042 Rennes, France \\ 5 Télécom SudParis, 9 Rue Charles Fourier, 91011 Evry, France \\ jacques.simonin@telecom-bretagne.eu, emmanuel.bertin@orange-ftgroup.com, yves.letraon@uni.lu, jezequel@irisa.fr,
} noel.crespi@it-sudparis.eu

\begin{abstract}
The main aim of Enterprise Architecture (EA) is to master the development and the evolutions of Information Systems (IS). The EA process consists in designing on several views the IS target architecture, according to the company strategy. The business view represents the target organization of the considered company. The functional view focuses on the target functional architecture of the considered IS. In this paper, we propose a new formal solution to analyze the consistency between the target functional view and the target business view of telecom services. This solution is based on the definition of a strategic alignment of the target functional view with the target business view. Alignment is illustrated with a real case study achieved with Orange - France Telecom on their messaging service. An alignment measure completing this analysis provides an estimation of the gap between a target functional view and a target business view.
\end{abstract}

Keywords - Information System, Enterprise Architecture, Business view, Functional view, Alignment, Measure.

\section{INTRODUCTION}

\section{A. Context and Motivation}

The Enterprise Architecture (EA) aims to simplify the Information Systems (IS) of a company, and to reduce the cost of IS development and evolution. This simplification of the IS should be driven by the strategy of the company. For telecommunication service providers, the strategy mainly consists in providing new services (designed by the marketing to fit user's needs) that rely as much as possible on existing infrastructures.

EA frameworks (like Zachman [1]) define various points of view (business, system, technology, etc.) in order to take into account all the aspects of these strategic objectives. This paper relies on the four classic EA views (defined for instance in [2]): the business view defining 'why', the functional view defining 'what', the technical view defining 'with what', and the applicative view defining 'how'. The relationships between the functional view, the technical view, and the applicative view are deduced from the iterative development cycle, which relies on the Unified Process (UP)
[3]. The business view should be an input for both the functional and the technical views.

This paper is focused on the strategic alignment of the functional view with the business view of a company. A good alignment highlights the consistency between the organization of the company and its IS [4]. It indicated that the business strategy and the IS strategy are synchronized.

The target business architecture and the target functional architecture have indeed both to fulfil the strategy of the company. However, the strategy concerning the business organization (business view) and the strategy concerning the IS functions (functional view) are different and are not defined by the same people. Business and functional views are evolving independently, following respectively the business and the marketing evolutions. An evolution of the company organization is moreover seldom synchronous with the evolution of its IS.

We propose thus an innovative formal approach that allows an enterprise functional architect to analyze the misalignment between the target functional architecture and the target business architecture. We propose moreover a metric of this alignment. The objective of this measure is to define an assessment in order to improve the alignment between the functional view and the business view.

\section{B. Outline}

This paper is organized as follows. Section II depicts state of the art and Section III introduces EA views and the alignment definition of the functional view with the business view. Section IV describes the alignment measure of the functional view with the business view. The example in Section III and in Section IV is based on Orange messaging service. Section V depicts the first experimentation of the alignment measure at the Orange Labs.

\section{RELATED WORK}

In the industry, most telecommunication companies directly map their business view with their IS applicative, in order to perform the alignment between their core business and their IS. So, a company may decide that a given email 
platform (for example, Microsoft Exchange server) will be in charge of the whole service business process of communicating by emails. As far as telecom services are concerned, this method has one main shortcoming: it implies a tight coupling between the business view and the applicative view. The business analysis is then distorted by applicative considerations. For example, the messaging business may become only driven by the evolutions of the selected platform, and no more by the company strategy. The specification of a target business architecture that differs from the current applicative view is hardly possible.

In the academic literature, the problem of the alignment involving EA is mainly considered between the business view of a company and its IS [5]. Alignment may be also considered between the business view of a company and its objectives as in the Business Motivation Model [6], or between an analysis model and a design model of the functional view of a telecom service [7]. The parameters related to the quality of the alignment are specific for each company [8]. For this kind of alignment, heuristics may be defined to provide warnings in case of misalignment [4]. Measurement method especially allows evaluating architectures in business terms (cost, benefit, risk). Nevertheless, measures in relation to business terms do not take into account IS concepts.

A contribution of this paper is to take into account the effects of the company strategy on the functional view. The alignment perspective between the business and functional views is shown in Figure 1.

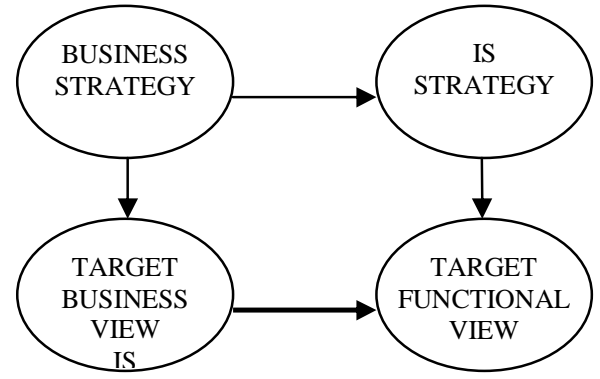

Figure 1. Alignment perspective between the target business view deduced from business strategy and the IS target functional view deduced from IS strategy [5].

The functional view choice is justified because IS functional view is easier to align with business view. Function meaning intelligibility is indeed helpful. IT applicative view moreover implements largely it. Alignment of the applicative view with the business view is thus dependent on the alignment of the functional view with the business view.

Moreover, many object-oriented measures exist outside the EA scope. To estimate models alignment in this paper, coupling measures [9] are the most appropriate because relationships between models are the main characteristic of the proposed solution.

\section{ALIGNMENT OF THE FUNCTIONAL VIEW WITH THE BUSINESS VIEW DEFINITION}

We focus here on the alignment or the misalignment of the functional view with the business view. As we have seen in the previous section, this topic is barely studied in the state of the art.

\section{A. EA and Target Architecture}

The EA process has two main goals:

- to depict existing IT architecture, in order to describe what functions are implemented on each IT system, how each IT system is deployed and, which process is supported by each IT system;

- to design several target architecture views, in order to separate the concerns of the various stakeholders in the enterprise.

Even if the company strategy is constant during the design of all the target architectures of these views, the needed skills are different: on one side, core business experts of the company elaborate the business view; on the other side, enterprise functional architects design the functional view. This independency is particularly significant for the evolution of each view because their lifecycles are different. A complete synchronization of the company organization evolutions and the IS evolutions is in fact very difficult to achieve for a large company. This is especially true for the telecom service operators, where markets are very dynamic.

The target business architecture of a company is usually elaborated following a process analysis, which enables to describe the business processes that belong to the core business of a company. The business view has for main concept the activity, which is a part of a business process and, which is under the responsibility of an organizational role. Concepts are modelled with UML [10] ones. An UML activity diagram can indeed be used to capture a procedure designed in the target business architecture. Within Orange France Telecom, the usage of telecom services is specified with about 10 roles and several tens of activities.

For illustration purpose, let us consider the messaging service limited to the message receipt. When a new requirement appears in the telecom operator strategy like the need to protect children from inappropriate electronic messages, the access control must evolve. In this example, the operator chooses to implement its strategy by creating a new Child protection provider role. Furthermore the Messaging service provider role will depend on the new Child protection provider role in the new target organizational infrastructure. So, to achieve the messaging receipt activity, the Messaging service provider role needs the Child protection provider role intervention.

The procedure deduced from the messaging service process is therefore easily captured using an activity diagram as illustrated in Figure 2. 


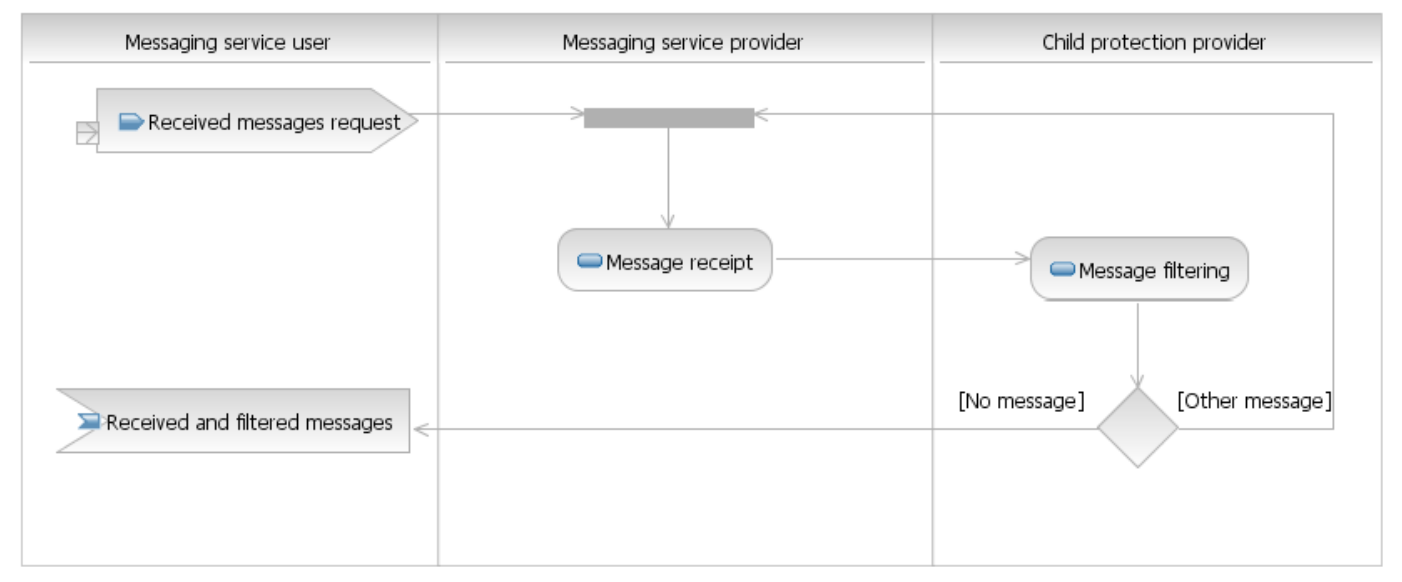

Figure 2. Sample activity diagram of messaging service.

The IS target functional architecture contains functional elements implemented by IS systems. Enterprise functional architects design the target functional architecture according to the company strategy. The main concept of the functional view metamodel is the function that defines functional component. Functional view concepts such as "Functional component" and "Dependency between functional components" are also closed to UML concepts. The target functional view may be represented by a component diagram. The target functional view of our messaging illustration is for example represented by the component diagram in Figure 3.

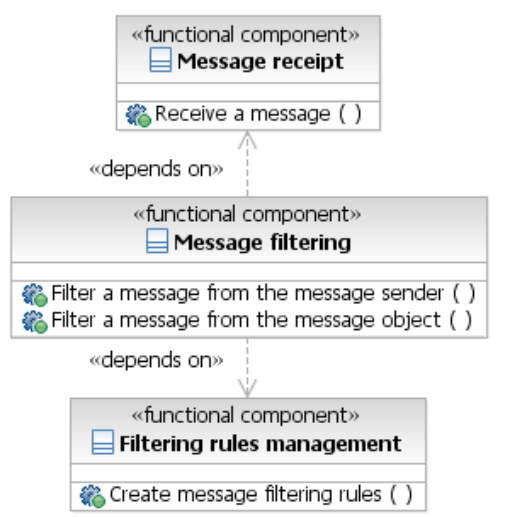

Figure 3. Sample target functional architecture of messaging service.

\section{B. Alignment of the Functional View with the Business View Definition}

Alignment criteria are required to define the alignment between models. Our innovative criterion is based on associations between concepts of the business view and of the functional view. Enterprise architect chooses business view concept consistent with functional view concept took into account into alignment definition. The consistency between these concepts means the alignment value between a business model and a functional model.

We have considered two relevant kinds of possible associations:

- associations between business data manipulated by business activities and functional data manipulated by functions.

- associations between business activities and functions.

An approach based on the first kind (business data and functional data) can be considered as static because it does not take into account the evolution of the states of data.

We have thus chosen an innovative approach by considering the second kind (business activities and functions). We have qualified this approach as dynamic, because it relies on the UML dynamic diagrams (activity diagram, sequence diagram) that show the live comportment of a system.

The idea of dynamic approach (as opposed to an approach based on data) is to base the alignment on service usage scenarios instead to base it on data models. For development methods in relation to the entity relationship model [11], the methodological complexity is a consequence of the simultaneous modelling of data and treatments. To resolve this complexity, our approach is based on the dynamic point of view because it allows functional reusability improving. This reusability involves service component called enablers as defined by OMA (Open Mobile Alliance) [12]. Moreover, the alignment between business data and functional data can be deduced from the alignment between business activities and functions, as business data are produced by business activities and functional data by functions.

The business view, as illustrated in Figure 2, instantiates dynamic concepts. A procedure is indeed described by an activity sequence instead of a business data model. Concerning the functional view, the design of an interaction sequence carrying out a telecom service usage scenario precedes indeed the data modelling. This chaining is feasible because each data is produced or used by a function during a 
scenario. With this dynamic approach, a dependency between functional components corresponds to an interaction between two functional component instances. The equivalence between an interaction sequence and a telecom service usage scenario denotes the dynamic aspect of the approach (see Figure 4).

So, a "request" type dependency of the functional view is an information request. A functional dependency has a "resource" type if it represents an answer to an information request.

The association completing the alignment criterion is between a succession relationship of two business activities and a dependency between functions. We define the following links:

- Succession relationship between two business activities if the end of one precedes the beginning of the other in an UML activity diagram capturing a business process (for example, in Figure 2, the succession relationship instantiated from the business activity Message receipt to the business activity Message filtering);

- Dependency between two functions

0 if they are associated to two interactions between functional components, which have either the "request" type or the "resource" type,

- and if the end of one of these interactions precedes the beginning of the other interaction in an UML sequence diagram

(an example in Figure 4 is the dependency between the function Filter a message from the message sender on the function Create message filtering rule).

The alignment of the functional view with the business view can thus be defined from these alignment criteria:

- a function is aligned with the business view

$\circ$ if the function has a common meaning with at least one activity of the business view,
- and if each aligned business activity with the function has at least one succession relationship with another aligned business activity with the function :

- a dependency between two functions F1 and F2, as $\mathrm{F} 1$ depends on $\mathrm{F} 2$, is aligned with the business view

$\circ$ if there is at least one business activity A1 aligned with $F 1$,

O if there is at least one business activity A2 aligned with $\mathrm{F} 2$,

- and if A1 follows A2 in an activity diagram (succession relationship).

The alignment definition is illustrated with the Figure 2 for the business activity and the activity succession relationship concepts, with the Figure 3 for the function concept, and with the Figure 4 for the function dependency concept:

- Receive a message function is aligned with

- Message receipt business activity,

- Filter a message from the message sender and Filter a message from the message object functions are aligned with

- Message filtering business activity,

- the dependency relationship from Filter a message from the message sender function on Receive a message function is aligned with

o the succession relationship from Message receipt business activity to Message filtering business activity

Create message filtering rule function and the dependency relationship from Filter a message from the message sender function on Create message filtering rule function, in Figure 4 , are not aligned with the business view. No business activity in Figure 2 has indeed a common meaning with Create message filtering rule function.

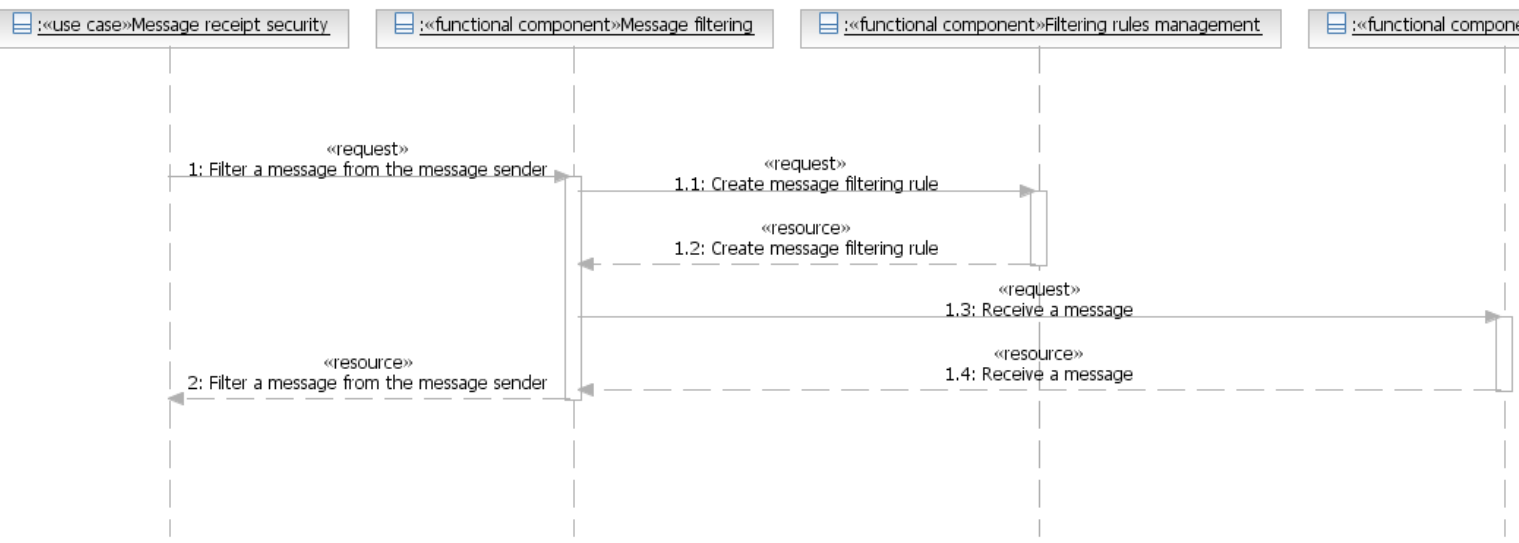

Figure 4. Functional view sequence of messaging service example. 


\section{AlignMENT OF THE FunCtional VIEW WITH THE BUSINESS VIEW MEASURE}

Axiomatization enables to specify the intuitive properties of the alignment of a functional view compared to a business view description [13]. We propose an alignment measure according to these axioms.

\section{A. Alignment of the Functional View with the Business View Axiomatization}

An axiom is an expected and understandable property of the alignment measurement that has also a meaning in the mathematical model. The following BFA axioms define this intuitive behaviour. Axioms are parameterized by functional view concepts concerned by the alignment.

BFA1 - Function addition. The alignment resulting from the addition of a function in the functional view is,

- worse than or identical to the previous alignment if the function has no common business meaning with at least one activity of the business view,

- better than the previous alignment if the function has a common business meaning with at least one activity of the business view.

BFA2 - Function dependency addition. The alignment resulting from the addition of a dependency between functions in the functional view is,

- worse than or identical to the previous alignment if there is no business activity time succession of the business view, which is aligned with the function dependency,

- better than the previous alignment if there is at least one business activity time succession of the business view, which is aligned with the function dependency.

BFA3 - Function deletion. The alignment resulting from the deletion of a function in the functional view is,

- worse than the previous alignment if the function has a common business meaning with at least one activity of the business view,

- better than or identical to the previous alignment if the function has no common business meaning with at least one activity of the business view.

BFA4 - Function dependency deletion. The alignment resulting from the deletion of a dependency between functions in the functional view is,

- worse than the previous alignment if there is at least one business activity time succession of the business view, which is aligned with the function dependency,

- better than or identical to the previous alignment if there is no business activity time succession of the business view, which is aligned with the function dependency.

\section{B. Alignment of the Functional View with the Business View Measure}

An alignment measure depends on alignment concepts defined in the Section III. The number of relationships captured in a diagram is a well known parameter for data model estimation [14]. The dependencies from the target functional view are the parameters of a proposed alignment measure, named BFAM, of the functional view with the business view. These dependencies, which are aligned with the business view or not, enable estimating the alignment of the functional view with the business view.

$$
\begin{aligned}
& \operatorname{BFAM}(F V)=\left(\frac{N_{-} f(F V)-N_{-}\{n a f\}(F V)}{N_{-} f(F V)}\right) * \\
& \left(\frac{N_{-} d(F V)-N_{-}\{n a d\}(F V)}{N_{-} d(F V)}\right)
\end{aligned}
$$

where, for a functional view $\mathrm{FV}$ :

- $\quad \mathrm{N} f(\mathrm{FV})$ is the number of functions,

- $\quad N_{-}\{$naf $\}(F V)$ is the number of functions, which are not aligned with business activities,

- $\quad \mathrm{N} \_\mathrm{d}(\mathrm{FV})$ is the number of dependencies between functions,

- $\quad N_{-}\{\operatorname{nad}\}(F V)$ is the number of dependencies between functions, which are not aligned with business activity time succession of the business view.

BFAM value is a real included between 0 (no function and no dependency relationship between functions are aligned with the business view) and 1 for a perfect alignment (all functions and all dependency relationships between functions are aligned with the business view).

BFAM measure complies with axioms BFA1, BFA2, BFA3, BFA4 (see Section IV) of the alignment of the functional view with the business view. Compliance is not detailed in this paper.

The alignment measure BFAM may be the stop criterion of an iterative development process. A higher estimation of the alignment means indeed a better consistency between target business view and target functional view.

\section{CASE STUdy}

The alignment measure of the functional view with the business view is a tool for functional architects in order to compare the business alignment of various functional domains (like messaging, IPTV, telephony...) and so to prioritize their actions in order to improve the alignment. Such action can be guided by an assessment of the alignment. Case study with telecom messaging functions has been led within Orange Labs. This domain contains actually 8 functional components, 12 functions, 16 function dependencies between functional components for 6 scenarios. The associated business view contains 3 activities and 2 activity time successions. 
The alignment measure BFAM of the messaging domain functional view with the business view of telecom service usage is estimated (see formula (1)):

$$
B F A M(\text { Messaging })=\frac{7}{8}
$$

Let us illustrate with a simple case how to improve the alignment. The assessment for messaging is the following one: the alignment of the functional view of the Messaging domain with the business process of message sending would be perfect (i.e., with a measure estimated to 1) if the dependency relationship

- from Transmit a message function defining Message exchange functional component

- $\quad$ on Store a message function defining Message storage functional component could be reversed (see Figure 5).

\begin{tabular}{|c|c|c|}
\hline $\begin{array}{l}\text { "functional component" } \\
\bigoplus \text { Message exchange }\end{array}$ & "depends on" & $\begin{array}{c}\text { "functional component" } \\
\boxminus \text { Message storage }\end{array}$ \\
\hline 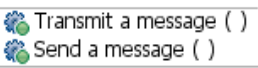 & & $\mathfrak{w}^{0}$ Store a message ( ) \\
\hline
\end{tabular}

Figure 5. Messaging domain problem

To keep aligned dependency from Send a message on Store a message, this reversal should require Message exchange functional component to be split as follows:

- Message transmission functional component defined by Send a message function,

- Message receipt functional component defined by Transmit a message function.

The dependency relationships represented in Figure 6 could provide thus a perfect alignment $($ BFAM $($ Messaging $)=1)$ :

- a dependency relationship from Message transmission functional component on Message receipt deduced from the Message exchange splitting,

- a dependency relationship from Message storage functional component on Message receipt improving the alignment,

- a dependency relationship from Message transmission functional component on Message storage resulting from the previous target functional architecture.

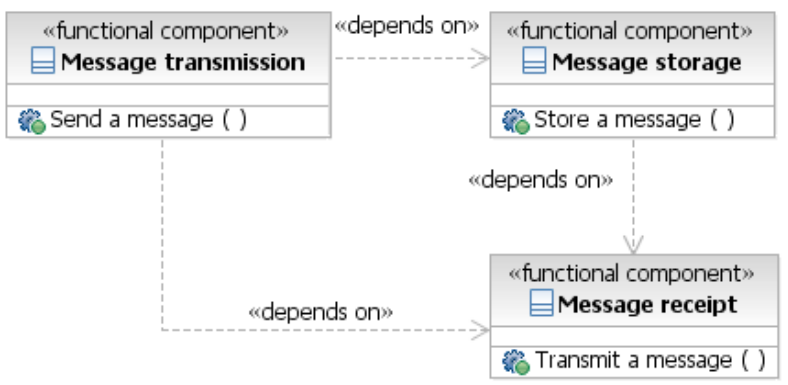

Figure 6. Messaging domain solution.
Functional architects may use this assessment as a tool to improve the business alignment, by checking if the suggested modifications are conform to the enterprise strategy.

\section{CONCLUSION}

The modelling process described in this paper enables to represent the alignment of an IS functional view with a business view of the IS owner company. The alignment definition is consistent with the meta-modelling from which business model and functional model are instantiated. An alignment measure is moreover proposed. This measure provides estimation from the synchronization of the company strategy integration for business and functional views.

Finally, a good alignment of the target functional view with the business target view induces a good alignment of the applicative view, which implements the target functional view, with the target business view. The applicative view contains several hundred of applications, which can hardly be directly mapped with the company business processes. In our approach, the target functional view is used as a link between the business and applicative views. This indirect mapping therefore allows an efficient tool to govern IT evolution according to the company strategy.

\section{REFERENCES}

[1] J.A. Zachman, "A Framework for Information Systems Architecture," IBM Systems Journal 26, no. 3, 1987, pp. 276-292.

[2] C. Longépé, "The Enterprise Architecture IT project - The Urbanisation paradigm,” Kogan Page, 2003.

[3] I. Jacobson, G. Booch, and J. Rumbaugh, "The Unified Software Development Process,” Addison-Wesley, 1999.

[4] C.M. Pereira and P. Sousa, "Getting into the Misalignment between Businesss and Information Systems," 10th European Conference on Information Technology Evaluation, Madrid, Spain, 2003.

[5] J.C. Henderson and N. Venkatraman, "Strategic Alignment: Leveraging Information Technology for Transforming Organizations," IBM Systems Journal, vol. 32, no. 1, 1993, pp. 4-16.

[6] The Business Rules Group, "The Business Motivation Model," http://www.businessrulesgroup.org/bmm.shtml, 2007.

[7] J. Simonin, Y. Le Traon, and J.-M.Jézéquel, "An Enterprise Architecture Alignment measure for Telecom Service development," 11th IEEE International Enterprise Distributed Object Computing Conference, Annapolis, Maryland U.S.A., 2007.

[8] J.N. Luftman, R. Papp, and T. Brier, "Enablers and Inhibitors of Buisness-IT Alignment," Communications of the Association for Information Systems, vol. 1, article 11, 1999.

[9] S.R. Chidamber and C.F. Kemerer, "Towards a Metrics Suite for Object Oriented Design," IEEE Transactions on Software Engineering, vol. 20, no. 6, 1994, pp. 476-493.

[10] G. Booch, J. Rumbaugh, and I. Jacobson, "The Unified Modeling Language - User Guide," Addison - Wesley, 1999.

[11] P.P.S. Chen, "The entity relationship model - Towards a unified view of data," ACM Transactions on Database Systems, vol. 1, no. 1, 1976, pp. 9-36.

[12] Open Mobile Alliance, "OMA Service Environment," OMA-ServiceEnvironment-V1_0_2-20050803-A, 2005.

[13] M.J. Shepperd and D. Ince, "Derivation and Validation of Software Metrics,” Oxford University Press, 1993.

[14] S.G. MacDonell, M.J. Shepperd, and P.J. Sallis, "Metrics for Database Systems: An Empirical Study," Software Metrics Symposium, 1997, pp. 99-107. 